\title{
Representations of the direct product of matrix algebras
}

\author{
by \\ Daniele Guido (Roma and Potenza) \\ and Lars Tuset (Roma and Oslo)
}

\begin{abstract}
Suppose $B$ is a unital algebra which is an algebraic product of full matrix algebras over an index set $X$. A bijection is set up between the equivalence classes of irreducible representations of $B$ as operators on a Banach space and the $\sigma$-complete ultrafilters on $X$ (Theorem 2.6). Therefore, if $X$ has less than measurable cardinality (e.g. accessible), the equivalence classes of the irreducible representations of $B$ are labeled by points of $X$, and all representations of $B$ are described (Theorem 3.3).
\end{abstract}

1. Introduction. Suppose $B=\prod_{x \in X} B\left(K_{x}\right)$ is an algebraic product of full matrix algebras $B\left(K_{x}\right)$ over an index set $X$. Clearly $B$ is a unital algebra under pointwise operations. Consider now a representation $\pi$ of $B$ on a Banach space. The purpose of this paper is to characterize such representations up to equivalence (see Theorem 3.3). A simple characterization is, however, only possible under the condition that $X$ is a set of less than measurable cardinality. While this condition imposes no real restriction on $X$ for practical applications of our result, since every set which can be constructed (by the ordinary operations in set theory, such as unions and powers) is of less than measurable cardinality, it is remarkable that such a nontrivial condition arises in this context. Theorem 3.3 says that every representation $\pi$ of $B$ (where the index set $X$ is assumed to be of less than measurable cardinality) is equivalent to a direct sum (including if necessary infinite multiplicity) of representations obtained by restricting $\pi$ to some of the factors $B\left(K_{x}\right)$ of $B$. As a corollary (see Corollary 3.4) to Theorem 3.3, we conclude that when $\pi$ is irreducible, it is equivalent to the representation of $B$ obtained by projecting down on one of the factors of $B$. We give a separate proof of this corollary as a shortcut for the reader. We note that

2000 Mathematics Subject Classification: Primary 46-XX; Secondary $03 E 55$.

Research of D. Guido supported by GNAFA and MURST.

Research of L. Tuset supported by CEE. 
our results do not depend crucially on the fact that we represent unbounded elements with bounded operators (cf. Remark 2.4).

The crucial notion in these investigations is that of a $\sigma$-complete ultrafilter. To explain how these filters occur, let $\pi$ be an irreducible representation of $B$ on a Banach space $K$. Define $F_{\pi}$ to be the collection of subsets of $X$ given by $F_{\pi}=\left\{U \in X \mid \pi\left(\chi_{U}\right)=I_{\pi}\right\}$. Here $I_{\pi}$ is the unit of $B(K)$ and $\chi_{U}$ is the element of $B$ defined by $\chi_{U}(x)=I_{x}$ for $x \in U$ and $\chi_{U}(x)=0$ for $x \notin U$, where $I_{x}$ is the unit of $B\left(K_{x}\right)$. We show (Lemma 2.3) that $F_{\pi}$ is a $\sigma$-complete ultrafilter over $X$. In fact, in Theorem 2.6 we establish our second main result, which states that the assignment $\pi \mapsto F_{\pi}$ induces a bijection between the equivalence classes of irreducible representations of $B$ on Banach spaces and the $\sigma$-complete ultrafilters over $X$. This result is true for an arbitrary index set $X$. However, it is known (see Lemma 2.5 and the paragraph preceding that lemma) that whenever $X$ is of less than measurable cardinality, then every such filter has to be principal. This way an irreducible representation $\pi$ of $B$ singles out a point in $X$ (over which the maximal principal filter is based), and the representation obtained by projecting down on the factor of $B$ corresponding to this point is equivalent to $\pi$.

We remark that in a very particular case our results are already known, namely when the spaces $K_{x}$ are one-dimensional. In this case $B$ is abelian, hence its representations are 1-dimensional. Therefore our results imply that all multiplicative linear functionals on $\mathbb{C}(X)$ are in $1: 1$ correspondence with $\sigma$-complete ultrafilters on $X$. As a consequence, all multiplicative linear functionals are given by Dirac measures if and only if $X$ has less than measurable cardinality. This result is contained in [1], where it was generalized in a direction different from ours: the fibers of the direct product were allowed to be arbitrary algebras over an arbitrary field (with some assumptions on the cardinality of the field) but only one-dimensional representations, namely multiplicative linear functionals, were studied $\left({ }^{1}\right)$.

Even this particular case cannot be easily guessed from analogous results. For instance, the set of characters of the algebra of complex-valued functions on $X$ with finite support (or vanishing at infinity) can be identified with the points of $X$. This is a particular case of the result that every character on the $C^{*}$-algebra of continuous complex-valued functions vanishing at infinity on a locally compact Hausdorff space is a Dirac measure (cf. [6]). If instead we consider the algebra of bounded complex-valued functions on $X$, then the set of characters is the Stone-Cech compactification of the space $X$ equipped with the discrete topology (cf. [2]). Thus in this case the characters are Dirac measures only when $X$ is finite. It is remarkable that passing from

$\left({ }^{1}\right)$ Indeed we became aware of [1] when this paper was already finished. 
bounded to unbounded functions amounts to passing from finite to less than measurable sets.

The idea of interpreting noncommutative algebras as noncommutative or quantum spaces has proved fruitful. We only mention the theory of noncommutative differential geometry developed by A. Connes (cf. [3]), and the theory of quantum groups (cf. [9]). In view of this one may conceive Corollary 3.4 as a first humble step towards "noncommutative set theory" with the role of "points" played by irreducible representations of the noncommutative product algebra $B$. Finally, notice that such product algebras appear in the theory of discrete quantum groups (cf. [10]). The index set is then the equivalence classes of a complete family of the dual compact quantum group, or if you like, the equivalence classes of irreducible objects in a concrete tensor $C^{*}$-category with conjugates (see [11]).

2. Filters and irreducible representations. Let $X$ be an arbitrary set, $x \mapsto K_{x}$ a map associating with any point $x \in X$ a Banach space $K_{x}$, and let $B\left(K_{x}\right)$ be the Banach algebra of bounded operators on $K_{x}$. Denote by $B$ the (unrestricted) algebraic direct product $B=\prod_{x \in X} B\left(K_{x}\right)$, i.e., $b \in B$ if $b$ is a function $b: X \rightarrow \bigcup_{x \in X} B\left(K_{x}\right)$ such that $b(x) \in B\left(K_{x}\right)$ for all $x \in X$. Clearly $B$ is a unital algebra with pointwise operations. Whenever the Banach spaces $K_{x}$ are in addition Hilbert spaces, the algebra $B$ is a *-algebra. When all the Banach spaces $K_{x}$ are the complex numbers $\mathbb{C}$, we denote the commutative unital algebra $B$ by $\mathbb{C}(X)$, which is all the complexvalued functions on the set $X$. A representation $\pi$ of $B$ on a Banach space $K$ is a unital homomorphism $\pi: B \rightarrow B(K)$. Two representations are equivalent if there is an invertible intertwiner between them. A representation is said to be irreducible if the only idempotents intertwining it are zero or the identity. If $K$ is a Hilbert space and $\pi(B)$ is a $*$-algebra (e.g. when $B$ is a $*$-algebra and $\pi$ is a $*$-representation), then the commutator $\pi(B)^{\prime}$ of $\pi(B)$ in $B(K)$ is a von Neumann algebra. Hence it is generated by its (self-adjoint) projections. Therefore irreducibility of $\pi$ is equivalent to the triviality of all (self-adjoint) projections intertwining it. However, when $\pi(B)$ is not a *-algebra (and $K$ is a Hilbert space), the two notions of irreducibility do not coincide as the following example shows.

Example 2.1. Consider $B=\mathbb{C}^{2}$. Denote by $\langle a\rangle$ the universal unital complex algebra generated by the element $a$ satisfying the relation $a^{2}=I$, where $I$ is the identity. Denote by $\langle b\rangle$ the unital universal complex algebra generated by the element $b$ satisfying the relation $b^{2}=b$. It is easy to see that $b \mapsto a=I-2 b$ extends to an isomorphism between $\langle b\rangle$ and $\langle a\rangle$. Now every element of $\langle b\rangle$ is of the form $\alpha I+\beta b$ for $\alpha, \beta \in \mathbb{C}$, thus $\langle a\rangle$ is isomorphic to $\mathbb{C}^{2}$. Let $M_{2}(\mathbb{C})$ be the unital complex algebra of $2 \times 2$-matrices with complex entries. Pick a number $h \notin\{0,1\}$, and define the $2 \times 2$-matrix 


$$
\pi(a)=\left(\begin{array}{cc}
0 & h^{-1} \\
h & 0
\end{array}\right) .
$$

We have $\pi(a)^{2}=I$, so by the universal property of $\langle a\rangle$ we get a representation $\pi: \mathbb{C}^{2} \rightarrow M_{2}(\mathbb{C})$. There are no nontrivial (self-adjoint) projections intertwining it. However, $\pi$ is not irreducible (in our stronger sense) as the (non-self-adjoint) idempotent $\frac{1}{2}(I-\pi(a))$ is nontrivial and intertwines $\pi$.

Lemma 2.2. Let $\pi: \mathbb{C}(X) \rightarrow B(K)$ be a representation, and let $\chi_{A} \in \mathbb{C}(X)$ be the characteristic function of $A \subset X$. If $e=\pi\left(\chi_{A}\right)$ is different from zero and the identity, then the spectrum $\sigma(e)$ of e equals $\{0,1\}$. If $f \geq 0$ for $f \in \mathbb{C}(X)$, then $\sigma(\pi(f)) \subset[0, \infty)$.

Proof. Suppose that $\lambda \notin\{0,1\}$. Then $\chi_{A}-\lambda I$ is invertible in $\mathbb{C}(X)$, so $e-\lambda I$ is invertible, and thus $\lambda \notin \sigma(e)$, showing that $\sigma(e) \subset\{0,1\}$. As $e \neq 0$ pick a nonzero vector $\xi \in e K \subset K$. Then $e \xi=\xi$, so $1 \in \sigma(e)$. And similarly, as $e \neq I$, pick a nonzero $\xi \in(I-e) K$. Then $e \xi=0$, so $0 \in \sigma(e)$, which proves the first assertion.

For the second assertion suppose first that $a>0$ and $-a \in \sigma(\pi(f))$. Then, by definition of spectrum, $\pi(f+a I)=\pi(f)+a I$ is not invertible. But $f+a I \in \mathbb{C}(X)$ is strictly positive, and therefore invertible, showing that $\pi(f+a I)$ is invertible, a contradiction. Therefore there are no (strictly) negative numbers in $\sigma(\pi(f))$. Next suppose that the imaginary part of $a \in$ $\sigma(\pi(f))$ is nonzero. Then we may write $a+1=\varrho \exp (i \theta)$ for numbers $\varrho, \theta$ with $\varrho>0$ and $\sin \theta \neq 0$. Thus by spectral calculus (see [8]), $-4 \sin ^{2} \theta<0$ belongs to

$$
\sigma\left(\pi\left(\left((f+I) \varrho^{-1}-(f+I)^{-1} \varrho\right)^{2}\right)\right)
$$

and clearly

$$
\left((f+I) \varrho^{-1}-(f+I)^{-1} \varrho\right)^{2} \geq 0,
$$

which is impossible according to the previous part of the proof. Therefore the imaginary part of $a \in \sigma(\pi(f))$ cannot be nonzero, nor can $a$ be negative, so $a \geq 0$, as desired.

Recall that a filter over a set $X$ is a collection $F$ of nonempty subsets of $X$ containing $X$, closed under finite intersections, and such that $V \in F$ whenever $U \in F$ and $U \subset V \subset X$. A filter $F$ is called an ultrafilter if for every $U \in X$ either $U \in F$ or its complement $U^{\mathrm{c}} \in F$. This happens if and only if $F$ is a maximal filter. A filter $F$ is called $\sigma$-complete if it is closed under countable intersections. More generally, if $\kappa$ is any regular uncountable cardinal (cf. [5]), we say that a filter $F$ is $\kappa$-complete if it is closed under intersections of less than $\kappa$ sets. It is known that an ultrafilter $F$ is $\sigma$-complete if and only if there is no countable partition $\left\{U_{n}\right\}_{n=0}^{\infty}$ of $X$ such that $U_{n} \notin F$ for all $n$. In fact, a similar result holds for $\kappa$-complete ultrafilters (see Exercise 27.2 in [5]), namely an ultrafilter is $\kappa$-complete if 
and only if for any partition of $X$ consisting of less than $\kappa$ elements, there exists a unique element of the partition belonging to the ultrafilter.

We will now see how an irreducible representation of $B$ gives a $\sigma$-complete ultrafilter over $X$. Let $U \subset X$ and define the element $\chi_{U} \in B$ by $\chi_{U}(x)=I_{x}$ if $x \in U$ and $\chi_{U}(x)=0$ if $x \in U^{\mathrm{c}}$, where $I_{x}$ is the identity element of $B\left(K_{x}\right)$. Let $\pi: B \rightarrow B(K)$ be an irreducible representation of $B$ on a Banach space $K$, and define $F_{\pi}$ to be the collection of subsets of $X$ given by

$$
F_{\pi}=\left\{U \subset X \mid \pi\left(\chi_{U}\right)=I_{\pi}\right\},
$$

where $I_{\pi}$ is the unit in $B(K)$.

Lemma 2.3. Let notation be as above. The collection $F_{\pi}$ is a $\sigma$-complete ultrafilter over $X$.

Proof. First note that $\pi(0)=0$ and $\pi\left(\chi_{X}\right)=\pi(I)=I_{\pi}$ assure that $F_{\pi}$ is a collection of nonempty subsets of $X$ containing $X$. If $U, V \in F_{\pi}$, then $U \cap V \in F_{\pi}$, because $\chi_{U \cap V}=\chi_{U} \chi_{V}$, so $\pi\left(\chi_{U \cap V}\right)=\pi\left(\chi_{U}\right) \pi\left(\chi_{V}\right)=I_{\pi}^{2}=I_{\pi}$. Let $U \subset X$. Note that $\chi_{U} b=b \chi_{U}$, so $\pi\left(\chi_{U}\right) \pi(b)=\pi(b) \pi\left(\chi_{U}\right)$ for all $b \in B$, and furthermore $\pi\left(\chi_{U}\right)^{2}=\pi\left(\chi_{U}\right)$ as $\chi_{U}^{2}=\chi_{U}$. Therefore $\pi\left(\chi_{U}\right)$, being an idempotent intertwining the (strongly) irreducible $\pi$, has to be either 0 or $I_{\pi}$. Let $U \in F_{\pi}$ and suppose that $U \subset V$. Then $V \in F_{\pi}$, because $\chi_{V}-\chi_{U}=\chi_{V \backslash U}$ and so

$$
\pi\left(\chi_{V}\right)-I_{\pi}=\pi\left(\chi_{V \backslash U}\right) \in\left\{0, I_{\pi}\right\},
$$

having $\pi\left(\chi_{V}\right)=I_{\pi}$ as the only solution within $\left\{0, I_{\pi}\right\}$. Thus $V \in F_{\pi}$ as well. If $I: x \mapsto I_{x}, x \in X$, is the unit of $B$, then $I-\chi_{U}=\chi_{U^{c}}$, and thus $I_{\pi}-\pi\left(\chi_{U}\right)=\pi\left(\chi_{U^{c}}\right)$ for any $U \subset X$. Hence $U \in F_{\pi}$ or $U^{\mathrm{c}} \in F_{\pi}$, because if $U \notin F_{\pi}$, then $\pi\left(\chi_{U}\right)=0$, meaning that $\pi\left(\chi_{U^{c}}\right)=I_{\pi}-\pi\left(\chi_{U}\right)=I_{\pi}$, and so $U^{\mathrm{c}} \in F_{\pi}$. We have thus shown that $F_{\pi}$ is an ultrafilter over $X$.

It remains to show that it is in fact $\sigma$-complete. Suppose by contradiction that we have a countable (disjoint) partition $\left\{U_{n}\right\}_{n=0}^{\infty}$ of $X$ such that $U_{n} \notin F_{\pi}$, i.e., $\pi\left(\chi_{U_{n}}\right)=0$ for all $n$. Define $b^{\infty}, b^{n} \in B$ by $b^{\infty}(x)=n I_{x}$ for $x \in U_{n}, n \in\{0, \ldots, \infty\}$, and $b^{n}(x)=k I_{x}$ for $x \in U_{k}, k \in\{0, \ldots, n\}$, whereas $b^{n}(x)=n I_{x}$ for $x \in U_{k}$ and $k \in\{n+1, \ldots, \infty\}$. Then

$$
b^{n}-n I=\sum_{k=0}^{n-1}(k-n) \chi_{U_{k}},
$$

and so

$$
\pi\left(b^{n}-n I\right)=\sum_{k=0}^{n-1}(k-n) \pi\left(\chi_{U_{k}}\right)=\sum_{k=0}^{n-1}(k-n) 0=0,
$$

which shows that $\pi\left(b^{n}\right)=\pi(n I)=n I_{\pi}$ for all $n$. But $b^{\infty}(x)=\alpha(x) I_{x}$ and $b^{n}(x)=\beta(x) I_{x}$ with $\alpha, \beta \in \mathbb{C}(X)$. Hence we may identify $b^{\infty}(x)$ with $\alpha$ and 
$b^{n}(x)$ with $\beta$, and using spectral calculus and Lemma 2.2, we deduce for all $n$ that

$$
\sigma\left(\pi\left(b^{\infty}\right)\right)-n=\sigma\left(\pi\left(b^{\infty}-b^{n}\right)\right),
$$

which is impossible, as the spectrum of any element in a Banach algebra is nonempty [8], and as Lemma 2.2 says, both $\sigma\left(\pi\left(b^{\infty}\right)\right)$ and $\sigma\left(\pi\left(b^{\infty}-b^{n}\right)\right)$ consist solely of positive numbers.

REMARK 2.4. The previous lemma is the key step which allows us to pass from ultrafilters to $\sigma$-complete ultrafilters, hence to $\gamma$-complete ones, $\gamma$ being the least measurable cardinal, as shown below. It is also the only place where the existence of unbounded elements in $B$ is used. Things do not change if we consider a Hilbert space $H$ and allow unbounded elements of $B$ to be represented by unbounded closed linear operators on $H$. Indeed, in this case the previous lemma also holds, hence again irreducible representations give rise to $\gamma$-complete ultrafilters.

Recall that a filter $F$ over $X$ is principal if there is a $V \subset X$ such that

$$
F=\{U \subset X \mid V \subset U\} .
$$

In case this $F$ is an ultrafilter, it is maximal, so $V$ has to be a one-point set. An uncountable set $X$ is of measurable cardinality if there exists a nonprincipal $X$-complete ultrafilter over it (cf. p. 297 in [5]). In particular the least cardinal (if any!) on which there is a $\sigma$-complete nonprincipal ultrafilter is measurable. We identify here the set $X$ with its cardinality. We shall say that a set has less than measurable cardinality if its cardinality is smaller than the first measurable cardinal.

Lemma 2.5. Let $F$ be a $\sigma$-complete ultrafilter over $X$. If $\gamma$ is the least measurable cardinal, then $F$ is a $\gamma$-complete ultrafilter over $X$. In particular, if $X$ has less than measurable cardinality, then $F$ is principal.

Proof. Let $\left\{X_{i}\right\}_{i \in \kappa}$ be a partition of $X, \kappa<\gamma$. We must show that there exists $j \in \kappa$ such that $X_{j} \in F$. Define a collection of subsets of $\kappa$ by

$$
F_{\kappa}=\left\{I \subset \kappa \mid \bigcup_{i \in I} X_{i} \in F\right\} \text {. }
$$

We claim that $F_{\kappa}$ is a $\sigma$-complete ultrafilter over $\kappa$ : Clearly $X \in F$ implies that $\kappa \in F_{\kappa}$, and $F_{\kappa}$ consists of nonempty subsets only. If $I, J \in F_{\kappa}$, then as $\left\{X_{i}\right\}_{i \in \kappa}$ is a partition of $X$, we have

$$
\bigcup_{i \in I \cap J} X_{i}=\bigcup_{i \in I} X_{i} \cap \bigcup_{i \in J} X_{i} \in F
$$

so $I \cap J \in F_{\kappa}$. If $J \in F_{\kappa}$ and $I \supset J$, then $I \in F_{\kappa}$ as $\bigcup_{i \in I} X_{i} \supset \bigcup_{i \in J} X_{i} \in F$. 
Furthermore, if $J \notin F_{\kappa}$ then $J^{\mathrm{c}} \in F_{\kappa}$, because

$$
\bigcup_{i \in J^{\mathrm{c}}} X_{i}=\left(\bigcup_{i \in J} X_{i}\right)^{\mathrm{c}}
$$

as $\left\{X_{i}\right\}_{i \in \kappa}$ is a partition. Finally, if $\left\{\kappa_{n}\right\}_{n=0}^{\infty}$ is a countable partition of $\kappa$, then $\left\{Y_{n}\right\}_{n=0}^{\infty}$ with $Y_{n}=\bigcup_{i \in \kappa_{n}} X_{i}$ is a countable partition of $X$. Thus there exists a number $m$ such that $\bigcup_{i \in \kappa_{m}} X_{i} \in F$, i.e., $\kappa_{m} \in F_{\kappa}$, so $F_{\kappa}$ is a $\sigma$-complete ultrafilter over $\kappa$. We conclude that $F_{\kappa}$ is a principal filter. Indeed, according to Lemma 27.1 of [5], the least cardinality for which there exists a nonprincipal $\sigma$-complete ultrafilter is measurable; therefore $F_{\kappa}$ is principal, hence there exists $j \in \kappa$ such that $X_{j} \in F$ as desired.

Now suppose that $0<\operatorname{dim} K_{x}<\infty$ for all $x \in X$. We will see how a $\sigma$-complete ultrafilter $F$ over $X$ together with a collection of bases, one for each $K_{x}, x \in X$, give rise to a finite-dimensional irreducible representation $\pi_{F}$ of $B$. Define for any natural number $n$ the set

$$
\Omega_{n}=\left\{x \in X \mid \operatorname{dim} K_{x}=n\right\} \subset X .
$$

Clearly, the collection $\left\{\Omega_{n}\right\}_{n=0}^{\infty}$ is a countable partition of $X$, and thus there exists a natural number $n(F)$ such that $\Omega_{n(F)} \in F$. This number $n(F)$ is also unique, since if any other member of the partition would belong to $F$, so would their intersection, which is empty, a contradiction. For any $M \in B\left(\mathbb{C}^{m}\right)$ we denote by $M_{i j}$ the matrix coefficients of $M$ with respect to the standard basis of $\mathbb{C}^{m}$. Let $b \in B$ and denote by $b(x)_{i j}$ the entries of the matrix $b(x)$ with respect to the chosen basis of $K_{x}$. Define $I_{M} \subset X$ by

$$
I_{M}=\left\{x \in \Omega_{n(F)} \mid b(x)_{i j}=M_{i j}\right\} .
$$

Note that $\Omega_{n(F)}^{\mathrm{c}}$ (which does not belong to $F$ ) together with the collection $\left\{I_{M} \mid M \in B\left(\mathbb{C}^{n(F)}\right)\right\}$ form a partition of $X$ into as many parts as there are real numbers. We now use the fact that a $\sigma$-complete ultrafilter is also $\gamma$-complete, where $\gamma$ is the least measurable cardinal (see Lemma 2.5). So in particular, for the partition above we know that there is a unique $M(b) \in$ $B\left(\mathbb{C}^{n(F)}\right)$ such that $I_{M(b)} \in F$. Define

$$
\pi_{F}(b)=M(b),
$$

and so we get a map $\pi_{F}: B \rightarrow B\left(\mathbb{C}^{n(F)}\right)$. We show that $\pi_{F}$ is indeed an irreducible representation of $B$ on $\mathbb{C}^{n(F)}$ : In proving this let us first agree on considering the operators as matrices with respect to the given bases, to avoid confusion with identifications. Then note that if $\Omega \in F$ and $b(x)=b(y)$ (as matrices) for all $x, y \in \Omega$, then $\pi_{F}(b)=b(x)$ for all $x \in \Omega$, because $\pi_{F}(b)=M(b)=b(x)$ for $x \in \Omega \cap I_{M(b)} \neq \emptyset$. Consider now $b_{1}, b_{2} \in B$ and form $I_{M\left(b_{1}\right)}$ and $I_{M\left(b_{2}\right)}$ in $F$ as described above. As $F$ is a filter, $\Omega=I_{M\left(b_{1}\right)} \cap I_{M\left(b_{2}\right)} \in F$, so $b_{i}(x)=b_{i}(y)$ for all $x, y \in \Omega$. Thus for 
$x \in \Omega$ it follows from the previous argument that

$$
\pi_{F}\left(b_{1} b_{2}\right)=\left(b_{1} b_{2}\right)(x)=b_{1}(x) b_{2}(x)=\pi_{F}\left(b_{1}\right) \pi\left(b_{2}\right) .
$$

Similarly one proves that $\pi$ is additive, and it is clearly unital. Finally, $\pi_{F}(B)=B\left(\mathbb{C}^{n(F)}\right)$ : indeed, if $M \in B\left(\mathbb{C}^{n(F)}\right)$, define $b \in B$ by

$$
b(x)= \begin{cases}M, & x \in \Omega_{n(F)}, \\ 0, & \text { otherwise. }\end{cases}
$$

Clearly $\pi_{F}(b)=M$, so $\pi_{F}$ is an irreducible representation. In fact, we have the following result.

TheOREM 2.6. Let $X$ be an arbitrary set. Suppose that the spaces $K_{x}$ are nonzero and finite-dimensional for all $x \in X$. The set of equivalence classes of irreducible representations of $B$ is in 1-1 correspondence with the set of $\sigma$-complete ultrafilters on $X$. More precisely, denote by $[\pi]$ the equivalence class of an irreducible representation $\pi$ of $B$ on a Banach space $K$, and let $\mathcal{E}$ be the set of all such equivalence classes (for all Banach spaces $K$ ). Denote by $\mathcal{F}$ the set of all $\sigma$-complete ultrafilters over $X$. Then the assignments

$$
[\pi] \mapsto F_{\pi}
$$

from $\mathcal{E}$ to $\mathcal{F}$ and

$$
F \mapsto\left[\pi_{F}\right]
$$

from $\mathcal{F}$ to $\mathcal{E}$, where $F_{\pi}$ is defined as in Lemma 2.3 and $\pi_{F}$ as above, are well defined, and they are inverse to each other.

Proof. The first assignment is well defined, as $F_{\pi}=F_{\pi^{\prime}}$ for two equivalent representations $\pi$ and $\pi^{\prime}$ of $B$. For the second assignment, notice that $\pi_{F}$ is defined using $F$ and a chosen collection of bases for each $K_{x}$. To prove that it is well defined, we therefore need to prove that $\left[\pi_{F}\right]$ is independent of this choice of bases. Say we have two collections (1) and (2) of bases for the spaces $K_{x}$ and form the two associated irreducible representations $\pi_{F}^{1}$ and $\pi_{F}^{2}$ of $B$ on $\mathbb{C}^{n(F)}$. We need to prove that they are equivalent. As (1) and (2) are collections of bases, we know that for each $x \in X$ there exists an invertible matrix $S(x) \in B\left(K_{x}\right)$ taking the basis in $K_{x}$ from (1) to the basis in $K_{x}$ from (2). For any (finite-dimensional complex) square matrix $S$ define the set

$$
X_{S}=\{x \in X \mid S(x)=S\} .
$$

The collection $\left\{X_{S}\right\}$ of subsets of $X$, where the index $S$ ranges over all quadratic matrices $S \in \bigcup_{n=0}^{\infty} \mathbf{M}_{n}(\mathbb{C})$, is clearly a partition of $X$ into no more parts than there are real numbers. As $F$ is a $\sigma$-complete ultrafilter, it follows from Lemma 2.5 that there exists a square matrix $S(F)$ such that $X_{S(F)} \in F$. Let $b \in B$ and consider the operators $\pi_{F}^{i}(B) \in B\left(\mathbb{C}^{n(F)}\right)$, $i=1$ or $i=2$, as matrices with respect to the standard bases (1) and (2). 
Furthermore, let $I_{M(b)}^{i} \in F$ be as in the definition of the representations $\pi_{F}^{i}$. Then as $F$ is a filter, we have

$$
\Omega=X_{S(F)} \cap I_{M(b)}^{1} \cap I_{M(b)}^{2} \in F .
$$

As noted just before Theorem 2.6, we thus conclude that for any $x \in \Omega$, with $b_{i}(x)$ the matrices with respect to the bases for $K_{x}$ from (i),

$$
\begin{aligned}
\pi_{F}^{2}(b) & =b_{2}(x)=S(x) b_{1}(x) S(x)^{-1} \\
& =S(F) b_{1}(x) S(F)^{-1}=S(F) \pi_{F}^{1}(b) S(F)^{-1} .
\end{aligned}
$$

Hence we have proved that $\pi_{F}^{1}$ and $\pi_{F}^{2}$ are equivalent, and thus that the second assignment in the theorem is well defined.

We now prove that these assignments are inverse to each other. If we start with $F \in \mathcal{F}$, form $\left[\pi_{F}\right] \in \mathcal{E}$ and $F_{\pi_{F}} \in \mathcal{F}$, then we clearly end up with $F$. Thus we only need to see that going in the other direction gives us the identity map on $\mathcal{E}$. Let $[\pi] \in \mathcal{E}$, and form $F_{\pi} \in \mathcal{F}$ as prescribed:

$$
F_{\pi}=\left\{U \subset X \mid \pi\left(\chi_{U}\right)=I_{\pi}\right\}
$$

where now $\pi$ is an irreducible representation of $B$ on $K$. To construct the irreducible representation $\pi_{F_{\pi}}$, we choose and fix a collection of bases for all the spaces $K_{x}$. Denote by $n(\pi)$ the natural number $n\left(F_{\pi}\right)$ associated with the $\sigma$-complete ultrafilter $F_{\pi}$ such that $\Omega_{n(\pi)} \in F_{\pi}$. Define $B_{n(\pi)}$ by

$$
B_{n(\pi)}=\prod_{x \in \Omega_{n(\pi)}} B\left(K_{x}\right)
$$

We complete the proof by showing that $\left[\pi_{F_{\pi}}\right]=[\pi]$, and we proceed in five steps:

First 1), we claim that

$$
\pi(B)=\pi\left(B_{n(\pi)}\right) .
$$

To see this notice that for $b \in B$, we have

$$
b=\chi_{\Omega_{n(\pi)}} b+\left(1-\chi_{\Omega_{n(\pi)}}\right) b
$$

thus $\pi(b)=\pi\left(\chi_{\Omega_{n(\pi)}} b\right)$ and $\chi_{\Omega_{n(\pi)}} b \in B_{n(\pi)}$.

Secondly 2$)$, obviously $B\left(\mathbb{C}^{n(\pi)}\right)$ equals

$$
C=\left\{b \in B_{n(\pi)} \mid \exists c, \forall x, b(x)_{i j}=c_{i j}\right\},
$$

when the operators are considered as matrices with respect to the bases for the spaces $K_{x}$ and $\mathbb{C}^{n(\pi)}$.

Thirdly 3), we have

$$
\pi\left(B_{n(\pi)}\right)=\pi(C) .
$$

For let $b \in B_{n(\pi)}$, define for $m \in B\left(\mathbb{C}^{n(\pi)}\right)$, with respect to bases for $K_{x}$ and $\mathbb{C}^{n(\pi)}$, the set

$$
X_{m}=\left\{x \in \Omega_{n(\pi)} \mid b(x)_{i j}=m_{i j}\right\},
$$


and consider the partition

$$
\left\{X_{m} \mid m \in B\left(\mathbb{C}^{n(\pi)}\right)\right\}
$$

of $\Omega_{n(\pi)}$. By adding $\Omega_{n(\pi)}^{\mathrm{c}} \notin F_{\pi}$ to this partition, we get a partition of $X$ into no more parts than there are real numbers. It then follows from Lemma 2.5 that there exists a unique $m(b)$ such that $X_{m(b)} \in F_{\pi}$. Then $\pi(b)=\pi(m(b))$, because

$$
\begin{aligned}
b-m(b) & =(b-m(b)) \chi_{X_{m(b)}}+(b-m(b))\left(1-\chi_{X_{m(b)}}\right) \\
& =(b-m(b)) \chi_{X_{m(b)}^{\mathrm{c}}} .
\end{aligned}
$$

Now $X_{m(b)}^{\mathrm{c}} \notin F_{\pi}$, so

$$
\pi(b-m(b))=\pi(b-m(b)) \pi\left(\chi_{X_{m(b)}^{c}}\right)=0 .
$$

From 1), 2) and 3) above we have $\pi(B)=\pi\left(B\left(\mathbb{C}^{n(\pi)}\right)\right)$. By nontriviality of $\pi$, we find that $\pi\left(B\left(\mathbb{C}^{n(\pi)}\right)\right)$ is isomorphic to $B\left(\mathbb{C}^{n(\pi)}\right)$, as otherwise ker $\pi$ would be a nontrivial ideal in the simple algebra $B\left(\mathbb{C}^{n(\pi)}\right)$ ). Thus $\pi(B)$ is isomorphic to the algebra $\left.B\left(\mathbb{C}^{n(\pi)}\right)\right)$, and as $\pi$ is irreducible, $K$ is isomorphic to $\mathbb{C}^{n(\pi)}$. We can now add the final step of the proof. By definition of the representation $\pi_{F_{\pi}}$ we know that for $b \in B$,

$$
\pi_{F_{\pi}}(b)=M(b),
$$

where

$$
\left\{x \in \Omega_{n(\pi)} \mid b(x)_{i j}=M(b)_{i j}\right\} \in F_{\pi} .
$$

But from the above, we see that

$$
\pi(b)=\pi(M(b))=M(b),
$$

by the identification of $\pi(B)$ with $B\left(\mathbb{C}^{n(\pi)}\right)$, so $\pi(b)=\pi_{F_{\pi}}(b)$ and $[\pi]=\left[\pi_{F_{\pi}}\right]$ as required.

REMARK 2.7. We may obviously generalize this results to the case where the spaces $K_{x}$ are Hilbert spaces with less than measurable dimension. More precisely, there is a 1-1 correspondence between equivalence classes of irreducible representations of $B$ (= algebraic product of the $B\left(K_{x}\right)^{\prime}$ 's for $x \in X$ ) and $\sigma$-complete ultrafilters over $X$.

3. Characterization of representations on $B$. Our main result in this section is Theorem 3.3. It says that whenever the index set over which the product in $B$ is taken is of less than measurable cardinality, then the only representations of $B$ on Banach spaces are finite sums of those one gets by projecting down on the factors in the product.

TheOREM 3.1. Let $X$ be a set of less than measurable cardinality, and let $\pi$ be a representation of $B$ on a Banach space $K$. Then $\pi$ is determined by the representations of the algebras $B\left(K_{x}\right)$ for a finite number of $x$ 's. More 
precisely, define for $Z \subset X$ the subalgebra $B_{Z}=\prod_{x \in Z} B\left(K_{x}\right)$ of $B$, and let $\pi_{Z}$ be the representation of $B$ on $K$ obtained by projecting down $B \rightarrow$ $B_{Z} \subset B$ and then restricting $\pi$ to $B_{Z}: \pi_{Z}(b)=\pi\left(\chi_{Z} b\right)$. Define $Y \subset X$ by

$$
Y=\left\{x \in X \mid \pi_{x} \neq 0\right\} .
$$

Then 1) $Y$ is a finite set, and 2) $\pi_{Y}$ is equivalent to $\pi$.

Proof. Define a collection $U_{\pi}$ of subsets of $X$ by

$$
U_{\pi}=\left\{U \subset X \mid \pi\left(\chi_{U}\right) \neq 0\right\} .
$$

The collection $U_{\pi}$ has the inclusion property, because if we had a set $A \in U_{\pi}$ and a set $B$ with $A \cap B=\emptyset$ such that $A \cup B \notin U_{\pi}$, then $0=\pi\left(\chi_{A \cup B}\right)=$ $\pi\left(\chi_{A}\right)+\pi\left(\chi_{B}\right)$, so $\pi\left(\chi_{B}\right)=-\pi\left(\chi_{A}\right)$, which is impossible as both $\pi\left(\chi_{A}\right) \neq 0$ and $\pi\left(\chi_{B}\right)$ are idempotents. But $U_{\pi}$ is not necessarily an ultrafilter (it is only when $\pi$ is irreducible). However, there exists a finite maximal partition $\left\{X_{i}\right\}_{i=1}^{n}$ of $X$ with the property that $\pi\left(\chi_{X_{i}}\right) \neq 0$ for all $i$, and furthermore, such that the collections

$$
F_{i}=\left\{U \in U_{\pi} \mid U \subset X_{i}\right\}
$$

are all $\sigma$-complete ultrafilters over $X_{i}$. Assume for the moment that we have such a partition of $X$. Observe first that $U \in U_{\pi}$ if and only if there exists $i$ such that $U \cap X_{i} \in F_{i}$. As $X$ is a set of less than measurable cardinality, so are the sets $X_{i}$, and so by Lemma 2.5 all filters $F_{i}$ are based on (unique) points $x_{i} \in X_{i}$. Thus $U \in U_{\pi}$ if and only if there exists $i$ such that $x_{i} \in U$. Now set $W=\left\{x_{i}\right\}_{i=1}^{n}$. Certainly $W$ is a finite subset of $X$. As $W^{\mathrm{c}} \cap X_{i}=X_{i} \backslash\left\{x_{i}\right\} \notin U_{\pi}$ and $X_{i} \in U_{\pi}$, it follows by the intersection property of $U_{\pi}$ that $W^{\mathrm{c}} \notin U_{\pi}$, so $\pi\left(\chi_{W^{\mathrm{c}}}\right)=0$. For $b \in B$ we thus get

$$
\pi(b)=\pi\left(\left(\chi_{W}+\chi_{W^{c}}\right) b\right)=\pi\left(\chi_{W} b\right)=\pi_{W}(b),
$$

so $\pi$ is equal (and so a fortiori equivalent) to $\pi_{W}$ and $W=Y$. Therefore we are done if we can prove that a finite partition $\left\{X_{i}\right\}_{i=1}^{n}$ of $X$ as described above does indeed exist. We first prove that if

$$
\text { there is a finite maximal partition }\left\{X_{i}\right\}_{i=1}^{n} \text { of } X
$$

$$
\text { such that } \pi\left(\chi_{X_{i}}\right) \neq 0 \text {, }
$$

then the collections $F_{i}$ are all $\sigma$-complete ultrafilters over $X_{i}$. To this end we first note that if $U \subset X_{j}$ with $\pi\left(\chi_{U}\right) \neq 0$, then $\pi\left(\chi_{U}\right)=\pi\left(\chi_{X_{j}}\right)$. This follows because

$$
\pi\left(\chi_{X_{j}}-\chi_{U}\right)=\pi\left(\chi_{X_{j} \cap U^{c}}\right)=0,
$$

as otherwise $\left\{X_{i}\right\}_{i \neq j}, U$ and $X_{j} \cap U$ would be a partition of $X$ satisfying (1) and strictly larger than the maximal one. Hence for any $U \subset X_{i}$, we have either $\pi\left(\chi_{U}\right)=0$ or $\pi\left(\chi_{U}\right)=\pi\left(\chi_{X_{i}}\right)$. Now proceed as in the proof of 
Lemma 2.3 with $I_{\pi}$ replaced by $\pi\left(\chi_{X_{i}}\right)$, to prove that the collections $F_{i}$ are all $\sigma$-complete ultrafilters over $X_{i}$.

Hence we are left with the task of proving the existence of a finite partition $\left\{X_{i}\right\}_{i=1}^{n}$ with property (1). As $\pi\left(\chi_{X}\right) \neq 0$, this is clearly equivalent to saying that

$\sup \left\{n \in \mathbb{N} \mid\right.$ there is a partition $\left\{X_{i}\right\}_{i=1}^{n}$ of $X$ such that $\left.\pi\left(\chi_{X_{i}}\right) \neq 0\right\}<\infty$. Using the inclusion property of $U_{\pi}$, one sees that the negation of this statement is equivalent to saying that

(2) for all $n \in \mathbb{N}$ there is a partition $\left\{X_{i}\right\}_{i=1}^{n}$ of $X$

$$
\text { with } \pi\left(\chi_{X_{i}}\right) \neq 0 \text { for all } i \text {. }
$$

We complete the proof by showing that property (2) leads to a contradiction. It suffices to construct a countable partition $\left\{X_{i}\right\}_{i=1}^{\infty}$ of $X$ such that $\pi\left(\chi_{X_{i}}\right) \neq 0$. Indeed, by Lemma 2.2 we then conclude that $1 \in \sigma\left(\pi\left(\chi_{X_{i}}\right)\right)$ for all $i$. Define $f \in B$ by $f=\sum_{n=1}^{\infty} n \chi_{X_{n}}$ and note that

$$
\sigma(\pi(f))=\bigcup_{n=1}^{\infty} n\left(\sigma\left(\pi\left(\chi_{X_{n}}\right)\right)\right) .
$$

Thus $\sigma(\pi(f))=\mathbb{N}$, which is a contradiction as the spectrum of an operator in a Banach space $B(K)$ is bounded.

Thus assume that property (2) holds for $X$. To construct the desired countable partition of $X$, we rely on the following property: if $U_{1}$ and $U_{2}$ form a partition of a set $U$ having property (2), then either $U_{1}$ or $U_{2}$ has property (2). Assume by contradiction that neither $U_{1}$ nor $U_{2}$ has property (2). Then there exist numbers $n_{1}, n_{2}$ such that $\left\{U_{j}^{i}\right\}_{i=1}^{n_{j}}$ are maximal partitions of $U_{j}$ such that $\pi\left(\chi_{U_{j}^{i}}\right) \neq 0$ for all $i=1, \ldots, n, j=1,2$. Let $N$ be $\max \left(n_{1}, n_{2}\right)$. Thus for $n>N$ there are no partitions $\left\{U_{j}^{i}\right\}_{i=1}^{n}$ of $U_{j}$ such that $\pi\left(\chi_{U_{j}^{i}}\right) \neq 0$ for all $i=1, \ldots, n, j=1,2$. Since $U$ has property (2), we may choose a partition $\left\{X_{i}\right\}_{i=1}^{2 N+1}$ of $U$ with $\pi\left(\chi_{X_{i}}\right) \neq 0$. Then define the sets $U_{j}^{i} \subset U_{j}$ by $U_{j}^{i}=U_{j} \cap X_{i}$. They give partitions of $U_{1}, U_{2}$, respectively, and, for any $i=1, \ldots, 2 N+1$ there exists $j$, depending on $i$, such that $\pi\left(\chi_{U_{j}^{i}}\right) \neq 0$, namely there exists $j=1,2$ such that at least $N+1$ elements of the partition $\bigcup_{i=1}^{2 N+1} U_{j}^{i}$ have the property that $\pi\left(\chi_{U_{j}^{i}}\right) \neq 0$. By adding the complement to one of the elements of the partition and using the inclusion property for $U_{\pi}$, we therefore obtain a partition $\left\{\Omega_{i}\right\}_{i=1}^{N+1}$ of one of the sets $U_{j}$ such that $\pi\left(\chi_{\Omega_{i}}\right) \neq 0$ for all $i$, contrary to the above. Thus either $U_{1}$ or $U_{2}$ has property $(2)$.

Consider now again the set $X$. It has property (2), so split it up in two parts, say $X_{1}^{0}$ and $X_{2}^{0}$ with $\pi\left(\chi_{X_{i}^{0}}\right) \neq 0$. Then by the above, (at least) one of them has property (2), say $X_{1}^{0}$, so we may split it up in two parts $X_{1}^{1}$ and 
$X_{2}^{1}$ with $\pi\left(\chi_{X_{i}^{1}}\right) \neq 0$. Again one of these must have property (2), say $X_{1}^{1}$, so we may split it up in two parts $X_{1}^{2}$ and $X_{2}^{2}$ with $\pi\left(\chi_{X_{i}^{2}}\right) \neq 0$, and one of them must have property (2) again, say $X_{1}^{2}$. This way we construct an infinite sequence $\left\{X_{1}^{i}\right\}_{i=1}^{\infty}$ of disjoint subsets of $X$ such that $\pi\left(\chi_{X_{2}^{i}}\right) \neq 0$. Now set

$$
X_{0}=\left(\bigcup_{i=1}^{\infty} X_{2}^{i}\right)^{\mathrm{c}} .
$$

Clearly $X_{0} \supset X_{2}^{0}$, so, by the inclusion property of $U_{\pi}$, we have $\pi\left(\chi_{X_{1}}\right) \neq 0$. For $i \geq 1$ define the sets $X_{i}=X_{2}^{i}$. Then clearly the collection $\left\{X_{i}\right\}_{i=0}^{\infty}$ is a countable partition of $X$ such that $\pi\left(\chi_{X_{i}}\right) \neq 0$ for all $i$. This concludes the proof.

We give the following lemma without proof, and refer to standard texts on $C^{*}$-algebras (cf. [4]).

Lemma 3.2. Let $A$ be a finite-dimensional $C^{*}$-algebra, so $A$ is isomorphic to a finite sum of full matrix algebras $\mathbf{M}_{k(i)}(\mathbb{C})$ :

$$
A \simeq \bigoplus_{i=1}^{m} \mathbf{M}_{k(i)}(\mathbb{C}) .
$$

Define the representations $\pi_{i}$ of $A$ on $\mathbb{C}^{k(i)}$ by

$$
\pi\left(\bigoplus_{j=1}^{m} m_{k(j)}\right)=m_{k(i)} .
$$

If $\pi$ is a representation of $A$ on a Banach space $K$, then there exist $B a$ nach spaces $K(i)$ such that $\pi$ is (weakly) equivalent to the representation $\bigoplus_{i=1}^{m} \pi_{i} \otimes I_{K(i)}$, where $I_{K(i)}$ is the identity operator on the Banach space $K(i)$.

Let $X$ be a set and suppose that all the Banach spaces $K_{x}$ in the product algebra $B$ are finite-dimensional. Denote by $p_{x}$ the representation of $B$ on $K_{x}$ obtained by projecting down on the factor $B\left(K_{x}\right)$, namely $p_{x}(b)=b(y)$. Then, given a finite subset $Y$ of $X$ and a Banach space valued map $\kappa$ : $x \in Y \mapsto K(x)$, we may consider the following representation of $B$ :

$$
p_{\kappa}=\bigoplus_{x \in Y} p_{x} \otimes I_{K(x)},
$$

$I_{K(x)}$ being the identity operator on the space $K(x)$. The following theorem is indeed a generalization (when the field of scalars is $\mathbb{C}$ ) of the main theorem in [1]. It may be further generalized to the case where the spaces $K_{x}$ are Hilbert spaces with less than measurable dimension (cf. Remark 2.7). 
THEOREM 3.3. Let $X$ be a set and suppose that all the Banach spaces $K_{x}$ in the product algebra $B$ are nonzero and finite-dimensional. Then the following two conditions are equivalent:

(a) $X$ has less than measurable cardinality.

(b) Any representation $\pi$ of $B$ on a Banach space $K$ is equivalent to $p_{\kappa}$ for some finite subset $Y$ in $X$ and some map $\kappa$ as above.

Proof. The implication $(\mathrm{a}) \Rightarrow(\mathrm{b})$ is an immediate consequence of Theorem 3.1 and Lemma 3.2. The implication $(\mathrm{a}) \Leftarrow(\mathrm{b})$ follows from Theorem 2.6.

The corollary below is an immediate consequence of Theorem 3.3. But as the proof of this theorem is rather long, we add a simplified proof of the corollary, from which the subsequent corollaries are proved as well.

COROLlary 3.4. Let $X$ be a set of less than measurable cardinality and suppose that all the Banach spaces $K_{x}$ in the product algebra $B$ are finitedimensional. Then any irreducible representation $\pi$ of $B$ is equivalent to $p_{y}$ for some $y \in X$.

Proof. Let $F_{\pi}$ be the $\sigma$-complete ultrafilter over $X$ given by the representation $\pi$ (see Lemma 2.3). According to Lemma 2.5, since $X$ has less than measurable cardinality, $F_{\pi}$ is principal. Thus for $X$ having less than measurable cardinality there exists $y \in X$ such that

$$
F_{\pi}=\{U \subset X \mid y \in U\} .
$$

For this $y \in X$, we find by definition of $F_{\pi}$ that $\pi\left(I_{y}\right)=I_{\pi}$, where we consider the unit $I_{y}$ of $B\left(K_{y}\right)$ as an element of $B$. As $I=I_{y}+I_{y^{\mathrm{c}}}$, we have $\pi\left(I_{y^{\mathrm{c}}}\right)=0$, and thus for all $b \in B$,

$$
\pi(b)=\pi(b I)=\pi\left(b\left(I_{y}+I_{y^{c}}\right)\right)=\pi(b(y))+\pi(b) \pi\left(I_{y^{c}}\right)=\pi(b(y)),
$$

where $b(y) \in B\left(K_{y}\right)$ is considered an element of $B$ and $I_{y^{c}}$ denotes the element $\chi_{\{y\}^{\mathrm{c}}} \in B$. Thus $b(y) \mapsto \pi(b(y))$ is a finite-dimensional irreducible representation of $B\left(K_{y}\right)$ on $K$. But the finite-dimensional algebra $B\left(K_{y}\right)$ is isomorphic to a matrix algebra, and we know (cf. for instance [4]) that every finite-dimensional irreducible representation of a matrix algebra is equivalent to the identity representation. Therefore there exists an invertible linear operator $S: K_{y} \rightarrow K$ such that $\pi(b(y))=S \circ b(y) \circ S^{-1}$ for all $b \in B$. Hence $\pi(b)=S \circ p_{y}(b) \circ S^{-1}$ for all $b \in B$, meaning that $\pi$ is equivalent to $p_{y}$, as desired.

REMARK 3.5. Let $X$ be a set of less than measurable cardinality. Consider the case where all the spaces $K_{x}$ are finite-dimensional, and endow them with a Hilbert space structure. Then since the representations $p_{x}$, 
$x \in X$, are all $*$-representations of $B$ on the Hilbert spaces $K_{x}$, every irreducible representation of $B=\prod_{x \in X} B\left(K_{x}\right)$ is equivalent to a (finitedimensional irreducible) *-representation of $B$ on some Hilbert space.

If we choose the Hilbert spaces in $B=\prod_{x \in X} B\left(K_{x}\right)$ all to be 1-dimensional, then an easy consequence of Theorem 2.6 and Corollary 3.4 is the result, due to [1] and mentioned in the introduction, that if $X$ is a set of less than measurable cardinality then all characters on $\mathbb{C}(X)$ are Dirac measures.

REMARK 3.6. We conclude this paper by observing that even though the theory of large cardinals is an active field of research concerning important set-theoretical questions, measurable cardinals are beyond any possibility of construction. In fact, the following results hold: Firstly, the existence of sets with measurable cardinality is inconsistent with the axiom of constructibility (cf. Section 31 of [5]). Secondly, measurable cardinals are all inaccessible cardinals (cf. Lemma 27.2 of [5]), which means for instance that their existence is not provable in ZFC (Zermelo-Fraenkel's set theoretical axioms and the axiom of choice); cf. Theorem 27 of [5]. Moreover, the same theorem, proved using Gödel's second incompleteness theorem, says that it cannot be shown that the existence of even inaccessible cardinals is consistent with ZFC. Therefore Corollary 3.4 holds for any "concrete" set $X$.

Acknowledgements. We thank Paolo Lipparini for conversations. We also thank the referee for calling our attention to reference [1].

\section{References}

[1] A. Białynicki-Birula and W. Żelazko, On the multiplicative linear functionals on the cartesian product of algebras, Bull. Acad. Polon. Sci. Cl. III 5 (1957), 589-593.

[2] N. Bourbaki, General Topology, Chapters 5-10, Springer, Berlin, 1980.

[3] A. Connes, Noncommutative Geometry, Academic Press, New York, 1994.

[4] J. M. G. Fell and R. S. Doran, Representations of ${ }^{*}$-Algebras, Locally Compact Groups, and Banach *-Algebraic Bundles, Academic Press, 1988.

[5] T. Jech, Set Theory, Academic Press, New York, 1978.

[6] G. J. Murphy, $C^{*}$-Algebras and Operator Theory, Academic Press, Boston, 1990.

[7] G. K. Pedersen, $C^{*}$-Algebras and their Automorphism Groups, Academic Press, London, 1979.

[8] W. Rudin, Functional Analysis, McGraw-Hill, New York, 1991.

[9] S. L. Woronowicz, Compact matrix pseudogroups, Comm. Math. Phys. 111 (1987), 613-665.

[10] -, Tannaka-Krein duality for compact matrix pseudogroups. Twisted $S U(N)$ groups, Invent. Math. 93 (1988), 35-76. 
[11] S. Yamagami, On unitary representation theories for compact quantum groups, Comm. Math. Phys. 167 (1995), 509-529.

Daniele Guido

Dipartimento di Matematica

Università di Roma "Tor Vergata"

I-00133 Roma, Italy

Present address:

Dipartimento di Matematica

Università della Basilicata

Contrada Macchia Romana

I-85100 Potenza, Italy

E-mail: guido@unibas.it
Lars Tuset

Dipartimento di Matematica Università di Roma "Tor Vergata" I-00133 Roma, Italy

Present address: Faculty of Engineering Oslo University College Cort Adelers Gate 30 0254 Oslo, Norway E-mail: larst@pc.iu.hioslo.no

Received 18 April 2000;

in revised form 23 January 2001 